\title{
Use of Blockchain in Healthcare: A Systematic Literature Review
}

\author{
Sobia Yaqoob ${ }^{1}$, Muhammad Murad Khan*2, Ramzan Talib ${ }^{3}$, Arslan Dawood Butt ${ }^{4}$, Sohaib Saleem ${ }^{5}$, \\ Fatima Arif ${ }^{6}$, Amna Nadeem ${ }^{7}$ \\ Department of Computer Science, Government College University Faisalabad, Faisalabad, Pakistan ${ }^{1,2,3,5,6}$ \\ Department of Computer Science and Information Technology, University of Okara, Okara, Pakistan ${ }^{1,5,7}$ \\ Department of Electrical Engineering, Government College University Faisalabad, Faisalabad, Pakistan ${ }^{4}$
}

\begin{abstract}
Blockchain is an emerging field which works on the concept of a digitally distributed ledger and consensus algorithm removing all the threats of intermediaries. Its early applications were related to the finance sector but now this concept has been extended to almost all the major areas of research including education, IoT, banking, supplychain, defense, governance, healthcare, etc. In the field of healthcare, stakeholders (provider, patient, payer, research organizations, and supply chain bearers) demand interoperability, security, authenticity, transparency, and streamlined transactions. Blockchain technology, built over the internet, has the potential to use the current healthcare data into peer to peer and interoperable manner by using a patient-centric approach eliminating the third party. Using this technology, applications can be built to manage and share secure, transparent and immutable audit trails with reduced systematic fraud. This study reviews existing literature in order to identify the major issues of various healthcare stakeholders and to explore the features of blockchain technology that could resolve identified issues. However, there are some challenges and limitations of this technology which are needed to be focused on future research.
\end{abstract}

Keywords-Issues; healthcare; blockchain; systematic review

\section{INTRODUCTION}

The blockchain technology was devised by an unidentified person "Satoshi Nakamoto" in October 2008. He proposed a peer-to-peer, non-intermediated, electronic cash system introducing the first digital currency named as Bitcoin. This Distributed Ledger Technology (DLT) is time-stamped chain of transactional blocks, sealed with a cryptographic hash function and digital signature implementing trustless protocol [1] [2] [3]. Bitcoin was the first application of blockchain technology implemented in 2009 [4], [5].

Blockchain (BC) is in its early stages but it captivated immense response and interest from the community, domain experts and researchers in various fields like banking, Internet of Things, manufacturing, governance, education, and healthcare (HC). Blockchain, distributed ledger technology, can contribute numerous benefits to the healthcare industry. Blockchain technology's diverse features like decentralization, immutability, robustness, security, privacy, currency and consensus via cryptographic algorithms [6], [4], [5]; has the capacity to address the current issues of medical and healthcare sector. It can alleviate reliance on a single centralized authority which is more vulnerable to inaccuracy and insecurity. The interoperable infrastructure of blockchain technology will enhance the data exchange among various healthcare peers to improve coordination, quality of care, innovations, and market competitions positively [7], [8], [9], [10]. This disruptive technology could have the potential to resolve the issues of counterfeit drugs, claims adjudication and patient billing management as it provides historical information to track the origin of transactions making all the actions transparent [9]. This technology can convert the current costly systems to costsaving or even money generating systems [11] as the users are rewarded with digital currency as an incentive for their contribution [10], [12], [13]. A blockchain based infrastructure can be envisaged for improved decentralized record keeping, health data exchange, reliable drug supply, claim processes, and costeffective systems. Blockchain has a tremendous potential to transform the current healthcare infrastructure. However, there are several challenges that have been identified and more research must be carried out to address these challenges such as scalability, security (threat of $51 \%$ attack), anonymity and fraud hype, disclosure of confidentiality, and environmental unsustainability.

This review paper explores how blockchain technology can revolutionize our current healthcare infrastructure. The sector comprises of different healthcare players (providers, payers, patients, vendors, manufacturers, and research institutes, etc.) performing different roles with different needs. This paper attempts to provide a deeper insight by discovering the scope of blockchain for all healthcare players in a single study and to identify the challenges of blockchain technology in the said sector.

This study is divided into six sections. Section I introduces the technology and its significance in the healthcare sector. Section II explores literature reviewed for this research which presents this trans-disciplinary study into following parts: first, it describes the blockchain technology and its features; second, it strives to find out studies highlighting most common issues of the healthcare sector and associated applications of blockchain technology in the sector; third, this work is compared with review papers to show the significance and need of this work. Section III narrates the research methodology comprising sections labeled as; the need of conducting a systematic literature review, motivation and research questions, search strategy, inclusion and exclusion criteria, classification criteria, and data extraction in accordance with the selected approach for conducting this systematic literature review. Section IV compiles the results to answer research questions heading to Section V which describes the probability of threats to the validity of this research. To end with Section VI, conclusion and future directions are documented for use of blockchain 
technologies in the healthcare sector.

The novelty of this work is that it will give insights to the readers to understand the features of this technology to develop and deploy the Blockchain based applications in healthcare sector. In this work, applications of blockchain technology are discussed in depth focusing all the healthcare players which will attract the readers to trace the maximum potentials of the technology in the healthcare sector. Furthermore, highlighted challenges of the technology described by this study will set directions for future research.

\section{LITERATURE REVIEW}

This section provides an overview of fundamental concepts related to the blockchain technology and several applications of this technology in the area of healthcare. Comparison of existing review papers has also been tabulated and discussed in this section.

\section{A. Blockchain}

Blockchain, a distributed ledger, is a chain of time-stamped blocks containing a specific number of validated transactions. Blocks are linked cryptographically using the hash value of the previous block. Each transaction generated by a user or node is digitally signed using a private key and broadcasted to the network. A validation/mining node takes up that transaction and encloses it into a block then block is broadcasted to the network [14]. Each node of the network checks the validation of the block by implementing the consensus protocol. The validated block is appended to the chain then updated ledger is replicated throughout the permissioned nodes of the network. Consensus protocol replaces the trusted third party or the central authority. Fig. 1 illustrates the difference between centralized and distributed ledger [1] [15] [16] [17]. The ledger provides security, auditability and anonymity-based transparency.
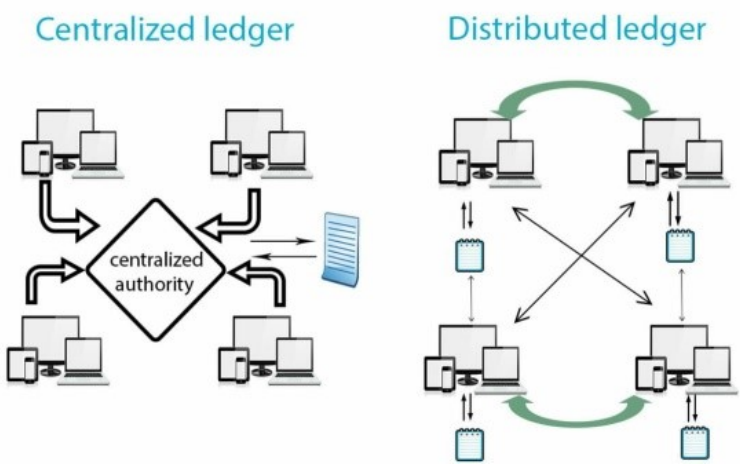

Fig. 1. Centralized vs. Distributed Ledger

1) Blockchain Evolution: Melanie Swan [15] categorizes the blockchain technology evolution into three phases: Blockchain 1.0, 2.0, and 3.0. Blockchain 1.0 is for the decentralization of money or known as "Internet of Money". This first application established a peer to peer digital payment systems without reliance on a third party. This tier of technology implements Proof of work, the consensus protocol for validating a block to embed in the ledger [4]. A digital reward is given to the successful miner for mining the block, for his contribution to the ledger. The second generation is blockchain 2.0 which is the application of decentralization of smart property and smart contracts, came in 2014. It aims to transfer any unit of value using the concepts of smart contracts for automated administration and supervision. The smart contract is a script which triggers after meeting the conditions encoded within it [17] [13]. Ethereum and Eris blockchains come under this category. The third blockchain technology refers to blockchain 3.0 which targets the welfare of society and is particularly recommended to register and transfer public records in the areas of government, health, science, literacy, and art. Examples are healthcoin, learningcoin, gridcoin, etc. [15]. Blockchain 2.0 and 3.0 are also known as non-financial applications. Alternative consensus protocols and alternative crypto-currencies have also been introduced.

2) Blockchain Ownership: There are two basic types of blockchain i.e. Permissioned blockchains and Permissionless blockchains. Permissioned blockchain is a custom-built setup by a single authority or a consortium. The verification process can be done by a central authority or a set of trusted preselected parties (consortium). This private setup restricts data access to the group of users or a set of groups that controls the blockchain. A smaller number of participants provides efficiency and scalability [18] [12]. These blockchains ultimately have a central authority. This centralization of the setup can pave way for tampering as the $51 \%$ majority is required to get the consensus and it can be done easily in this controlled setup [5]. Eris, Ripple, and Hyperledger are examples [1]. Permissionless blockchains are fully decentralized to a large number of nodes and low in efficiency [1]. These blockchains require no prior authorization of participants for mining the transaction blocks. Anyone can contribute his/her computational power for network tasks and can get a monetary reward in return. This blockchain gives the public access to read and write transactions to the blockchain which is visible to everyone so also known as Public blockchains [18] [12]. Examples of permissionless blockchains include Bitcoin and Ethereum [4].

3) Features of blockchain technology:

Decentralization: The blockchain is a distributed digital ledger composed of a chain of blocks containing transactions. The decentralized database is shared and open to all parties throughout the nodes of the network [6], [19], [20], [12]. BLockchain-based networks provide fault-tolerant architecture as end-to-end replications remove the reliance on a single point of failure.

Consensus mechanism: Blockchain is a peer-to-peer distributed network without any intermediary. Each digitally signed block is sent to the mining pool where it is taken over by network nodes called miners and verified using the consensus algorithm [4]. The winner from the miners broadcast the block to all other nodes which confirm and validate the block with consensus and append the block in their ledger. The winner also gets a financial reward for its work [21]. Many alternative consensus protocols have also been proposed, such as proofof-stake, proof-of-burn, proof-of elapsed-time [6], [12], [17]. Data integrity is maintained by computing these consensus algorithms as a substitute to third trusted party.

Immutability: Blockchain is immutable and tamper-proof thus ultimately provides security [15]. The hash function 
makes the blockchain as a tamper-resistant ledger. A hash value is calculated by implementing some hashing algorithms (SHA-256, RSA, RIPEMD-160, etc.) on a block of transactions [20]. The hash value is further used to create a chain of blocks. In this way blockchain provides robustness. If someone intends to alter previous transactions, then it will require a change in the hash value which further needs the consensus of network and high computational power which is an unrealistic approach in this computational model. The hash value is also used to represent a user concealing real identity which is used for privacy purposes [17], [22].

Traceability: Blockchain is a digital ledger consisting of continuously growing sequence of blocks. A block is comprised of a complete list of transaction records. In this chain of blocks, every block has a parent block. The first block in the chain is known as the genesis block. Hash code of genesis block is added to the header of the second block then hash code for the second block is computed over the hash of genesis block and transactions of the block jointly. Hash of the second block becomes the block header of the third block and so on. In this way, the blocks are linked with each other having a time stamp as well. This link can be chased back to the origin or genesis block [1], [20]. This feature of blockchain provides data provenance to keep chronical track of activities and may also help to investigate backward throughout the chain.

Smart Contract: A smart contract is a computerized computational logic or terms of the contract. It automatically triggers transactions between parties after fulfilling encoded logic. This implementation makes the blockchain flexible and programmable [1], [19] [13]. Smart contracts are programmed for management and administration purposes [6]. The smart contract can be implemented in supply chains, claim insurance [6] and clinical trials. Clinical trials usually encompass a sequence of dependent phases to get specific outcomes. Each phase can be encoded in a smart contract which will be triggered after the consensus of network nodes [23], [22]. So, smart contracts can enforce traceability and transparency with full control over associated processes.

Open Source: Blockchain projects are mostly open source. Developers can make contributions to it. Blockchain technology has the potential to accommodate the evolution to be brought by the future [8]. Transformation of financial blockchain to non-financial block-chains has already been announced in the big interest of the community. Ethereum and Counter-party have profound interest to build up more value-added services for the future architecture of blockchain technology [15].

Currency: The Bitcoin blockchain was first implemented in peer to peer digital payment system which also provides rewards in digital currency to the users for their contribution i.e. Proof of Work, to the network [6], [4]. Bitcoin was the first digital currency. In this evolution, many alt-crypto-currencies have been springing up. The major alternative currencies (altcurrencies) are monitored at http://coinmarketcap.com/. Over 1597 crypto-currencies have been developed in the digital cash system (Bitcoin, ethereum, ripple, litecoin, etc.). In different fields of life an economic layer can be embedded to give rewards in response to digital asset contribution and use e.g. learningcoin in education systems, journalcoin for the contribution of reviewers and editors, healthcoin to get national health services, etc. [15].

\section{B. Use of Blockchain in Healthcare}

Healthcare sector always remains one of the most popular areas of research from the last few decades keep on finding innovative and more reliable ways to help the community and healthcare industry. Different stakeholders (practitioners, medical specialists, hospitals, therapists, patients, payers, etc.) need to organize, access and share health records without any modification in a secure and interoperable way. Data provenance is also essential to prove the authenticity of records. Blockchain technology is being implemented in different scenarios and has the potentials to address the key issues of the healthcare sector. However, it needs more research to be focused to deploy real-time applications of this technology. Following are some applications of this technology in the healthcare sector.

MedRec platform provides decentralized record management, authorization and data sharing among healthcare stakeholders. Patients can save their data and can also grant and withdraw permissions to their records. This framework provides full confidentiality as the records are not stored on blockchain instead pointers to the data storage locations, logs and permissions are only stored in this blockchain [24], [4]. Gem in collaboration with Philips Blockchain Lab has been introduced Gem Health Network using Ethereum blockchain. This framework is established to address operational costs. This shared infrastructure provides interoperability among various providers accessing the same information to boost better patient care [8]. Guardtime healthcare platform creates a nonintermediated relationship between patient and provider in Estonia. Guardtime blockchain enabled transparent information sharing among the patient, provider, and payer which promises secure, reliable and auditable records [8]. Patient's health data is being required by research organizations. In this context, Healthbank has been providing a platform for patients to save and share their health data with research organizations which can be used for academic research and pharmaceuticals. This platform is also incentivizing patients with financial rewards for their contributions [8]. [25] designed Blockchain based Data Sharing (BBDS) access control system using permission blockchain. Owners of data can access their EMRs from a shared data pool. This secure and scalable system identifies, authenticates and authorizes users using cryptographic keys and digital signatures acquiring an edge over HDG (Healthcare Data Gateways) which is a smartphone application built over blockchain cloud [26]. Fast Healthcare Interoperability Resources: FHIRchain [27] was developed by the Health Level Seven International (HL7) organization for exchanging clinical data. FHIR increases efficiency and interoperability.

\section{Compared Secondary Studies}

Blockchain has been appeared a decade before in computing while it first came in the healthcare sector in 2014 with the advent of the non-financial version of the technology. Researchers are found enthusiastic to explore this unique technology to know the potentials and challenges. In this regard, six secondary studies were found that discuss the implementation of this technology is the healthcare sector. 
(IJACSA) International Journal of Advanced Computer Science and Applications,

TABLE I. COMPARED SECONDARY STUdIES

\begin{tabular}{|c|c|c|c|c|c|c|}
\hline \multicolumn{7}{|c|}{ Discussion Points } \\
\hline References & $\begin{array}{c}\text { Features of } \\
\text { BC }\end{array}$ & $\begin{array}{l}\text { Benefits of BC in HC } \\
\text { (All Stakeholders) }\end{array}$ & $\begin{array}{c}\text { Challenges and Issues to } \\
\text { BC Implementation }\end{array}$ & $\begin{array}{l}\text { BC Applications } \\
\text { in } \mathrm{HC}\end{array}$ & $\begin{array}{c}\text { Research } \\
\text { Methodology }\end{array}$ & $\begin{array}{l}\text { Cloud-based } B C \text { applications } \\
\text { and platform in } \mathrm{HC}\end{array}$ \\
\hline $\begin{array}{l}\text { T.Kuo et.al. [6], K. Rabah [9], } \\
\text { S. Angraal [28] }\end{array}$ & Partially Yes & No & Yes & Partially Yes & No & No \\
\hline M. Mettler [8] & Partially Yes & No & No & Partially Yes & No & No \\
\hline M. Benchoufi et. al. [23] & Partially Yes & No & No & Partially Yes & No & No \\
\hline D. Randall et. al. [29] & Partially Yes & No & No & No & No & No \\
\hline Y.Sobia et. al. & Yes & Yes & Yes & Yes & Yes & Yes \\
\hline
\end{tabular}

Table I presents five different aspects which have been reviewed by existing researchers and also compares to this study.

The first aspect in Table I is "features of blockchain technology". All the secondary studies partially narrate the features of blockchain as compared to this research. The second aspect discussed by earlier studies is "benefits of the blockchain technology in healthcare". It is extracted that M. Mettler [8], K. Rabah [9], as well as this work, made an analysis of this aspect with respect to all the stakeholders of healthcare sector whereas other four review papers discussed few of the stakeholders. The third mentioned aspect, Challenges and Issues to $\mathrm{BC}$ Implementation, has been highlighted by T. Kuo et al. [6], K. Rabah [9], S. Angraal [28] and this work whereas M. Mettler [8], M. Benchoufi et al. [23] and D. Randall et al. [29] did not address this aspect of concern.

Rapid development of this technology is being observed in healthcare market however after comparing these reviews, it is found that few of primary studies have been included on the subject of the fourth aspect, BC Applications in HC, except D. Randall et al. [29]. Whereas this study classifies more primary studies to analyze Blockchain Applications in the healthcare sector. Furthermore, qualitative research methodology has been opted to extract results in existing research. Hence, we used a quantitative research approach making a distinction to nonstructured review process i.e. systematic literature review, to classify the primary studies to make a deeper enclosure of all the aspects compared in Table I.

\section{Research Methodology}

A systematic literature review (SLR) is a type of review or secondary study which firstly defines specific research questions and then uses a well-defined methodology to collect, classify and extract all existing research to answer those questions [30] [31] [32]. Various guidelines are available for writing a Systematic Literature Review. However, the steps recommended by Barbara Kitchenham [30] [31] [32] are followed to conduct current research. This methodology is being followed by review papers published in high impact factor journals [33] [34]. This process has been formulated specifically for conducting systematic reviews for computing research.

\section{A. Need of Conducting SLR}

Table I presents the aspects discussed by existing research in accordance with the scope of this study. After analyzing earlier review papers, it is concluded that some of the aspects have been discussed briefly or ignored so far. Therefore, we focused to elaborate on the features of this technology to trace the maximum potentials in the healthcare sector. Furthermore, applications of blockchain technology are needed to be discussed in depth focusing all the healthcare players to fully transform the system. Thirdly, this systematic literature review executes a detailed and specialized sequence of activities to extract results which make the distinction to previous nonstructured reviews.

\section{B. Research Question $(R Q)$ and Motivation}

In response to the first phase of systematic literature review, the following research questions are formulated to cover the gaps found, as shown in Table II.

TABLE II. RESEARCH QUESTION AND Motivation

\begin{tabular}{c|c|c}
\hline Sr. \# & Research Question & Motivation \\
\hline 1 & $\begin{array}{c}\text { What are the major issues pertaining to } \\
\text { the Healthcare Stakeholders? }\end{array}$ & $\begin{array}{c}\text { The objective is to highlight major } \\
\text { issues obstructing the success of } \\
\text { Healthcare sector. }\end{array}$ \\
\hline 2 & $\begin{array}{c}\text { What Blockchain features are used to } \\
\text { resolve the identified issues? }\end{array}$ & $\begin{array}{c}\text { The aim is to explore the emerging } \\
\text { technology that resolves the } \\
\text { pertinent issues and accelerate the } \\
\text { said field. }\end{array}$ \\
\hline 3 & $\begin{array}{c}\text { What are the challenges and issues to } \\
\text { Blockchain implementation? }\end{array}$ & $\begin{array}{c}\text { Blockchain implementation issues } \\
\text { that are still unaddressed. }\end{array}$ \\
\hline
\end{tabular}

\section{Search Strategy}

Pursuing research questions, the following search queries were put to collect maximum literature for review:

"Issues in the Healthcare sector",

"Blockchain",

"Healthcare Blockchain"

"Systematic Review".

After careful exploration of different databases and journals, two hundred twenty-seven studies were collected. 


\section{Inclusion and Exclusion Criteria}

During this phase, some studies were found to be exactly aligned with the research area i.e. Blockchain and Healthcare, while others were found to be partially or completely out of context. Inclusion criteria limited the studies to thirty-nine since their titles and mentioned keywords were found to be similar to searched keywords. Studies published by famous publishers and impact factor journals were included. First of all, we analyzed the abstract of each shortlisted study according to research questions, methodology and findings of these papers and categorized them accordingly. Remaining searched articles were excluded because they did not include searched keywords in their titles and abstracts. Duplicate, irrelevant studies and publications are written in other than the English language were also excluded.

\section{E. Classification Criteria}

The aim of this research is to analyze existing research work implementing applications of blockchain technologies in the healthcare sector. Shortlisted studies were classified according to research questions.

\section{F. Data Extraction}

After classifying all studies to be included in the systematic review, the next step was to extract and analyze the information contained in those studies. Data extraction tables were designed using the spreadsheet to collect all information needed to address research questions i.e.

- Issues in Healthcare

- Features of Blockchain addressing those issues

- Challenges of Blockchain implementation.

Data extraction tables were filled in for each included study; further, those tables were used to accumulate the information.

\section{RESUlTS}

This section provides the details about research questions discussed under section III and further divided into three subsections. Section A identifies common issues of healthcare sector classified under different players of the said sector. Section B identifies blockchain features that can solve the current issues of the healthcare sector. Section $\mathrm{C}$ classifies the studies which highlight the issues and challenges of blockchain implementation which are to be fixed in the future.

\section{A. RQ1: What are the major issues pertaining to Healthcare Stakeholders?}

A system is a combination of some elements or objects which work together to make an effective output. Healthcare Sector is composed of five important players forming the ecosystem i.e. providers, patients, payers, supply chain bearer (manufacturers, vendors, pharmacy) and research organization. Fig. 2 provides an overview of the relationship between five major stakeholders in the healthcare sector. Some of the common relationships (triangles) are being identified in the following diagram where a common color of entities describes the relationship among them. Provider (hospital, doctor, specialist, etc.) plays a key role and usually acts as a third party. Each player is being encountered by some issues which are major areas of concern. These issues are discussed below under each scenario:

Scenario 1. Providers: A provider is a primary player in the healthcare sector. Patient record management is essential for successful outcomes for providers and patients. But there are numerous challenges to manage and curate the patient records as mentioned in Table III. Implementation of Electronic Medical Records (EMRs) and Electronic Health Records (EHRs) is one of the major issues confronted by healthcare providers as it is analyzed to be costly in terms of time and money both. Administrative costs of the Medicaid incentive program are estimated at approximately $\$ 30$ Billion i.e. $56 \%$ of total spend. It also includes deployment, maintenance and administrative costs [35], [36], [37]. The other major issue is fragmented patient records which increase poor communication among medical staff and a decrease in quality of care. Lack of interoperability standards to exchange health data among laboratories and hospitals is liable to higher overheads in time and resources [36] [38]. Furthermore, current document-centric and legacy IT systems in the healthcare sector are being run into a major issue relevant to the security and privacy of patients' data. Health data, maintained by a trusted third party in centralized databases, can be accessed by any malicious party, hacker, insider, or outsider [20], [16]. Providers act as a trusted third party for patients, payers, and pharmacies as shown in Fig. 2.

Scenario 2. Patients: A patient is the most important entity of the healthcare ecosystem. Patient health data is recorded by providers however patients do not have the rights to grant and revoke access to their medical records as per their desire [39]. The HIPAA (Health Insurance Portability and Accountability Act) Privacy Rules put restrictions on the usage of such information of patient's health. Patients are mostly concerned about the protection of their health data but their privacy is victimized in many ways. It is compromised when organization encrypts and decrypts the data. The other sources of collection of healthrelated personal data may include mobile apps, wearables, smart devices, etc. Social media networks are also found to be observed to collect users' personal data, actions, and habits without users' consent. Many private organizations collect, analyze and sell patients' personal health data to different companies for commercial benefits. Data may be used for drug marketing, research, public health care or some objectionable purposes [40], [41], [16]. Sharing of such personal data without the consent of a patient is the violation of HIPPA Privacy Rules [7], [3], [22]. Moreover, trusted third party is getting monetary benefits by selling patients' digital assets to outsiders as shown in Fig. 2 by using green color. Fragmented silos of patient health records or non-interoperability also leverage lack of communication between healthcare teams which results in poor quality of care, more time consumption and higher cost of reinvestigations [42].

Scenario 3. Payers: Insurance claim payments on behalf of patients by the payer (insurer or employer) are also needed to be verified from centric IT systems which are highly vulnerable to insecurity and intended fraudulent alterations [43]. Records (bills and prescriptions etc.) may be falsified by fake medical practitioner credentials, misbilling, and bogus testing, etc. Auditing and data provenance is crucial to track genuine insurance claim processes [44]. 


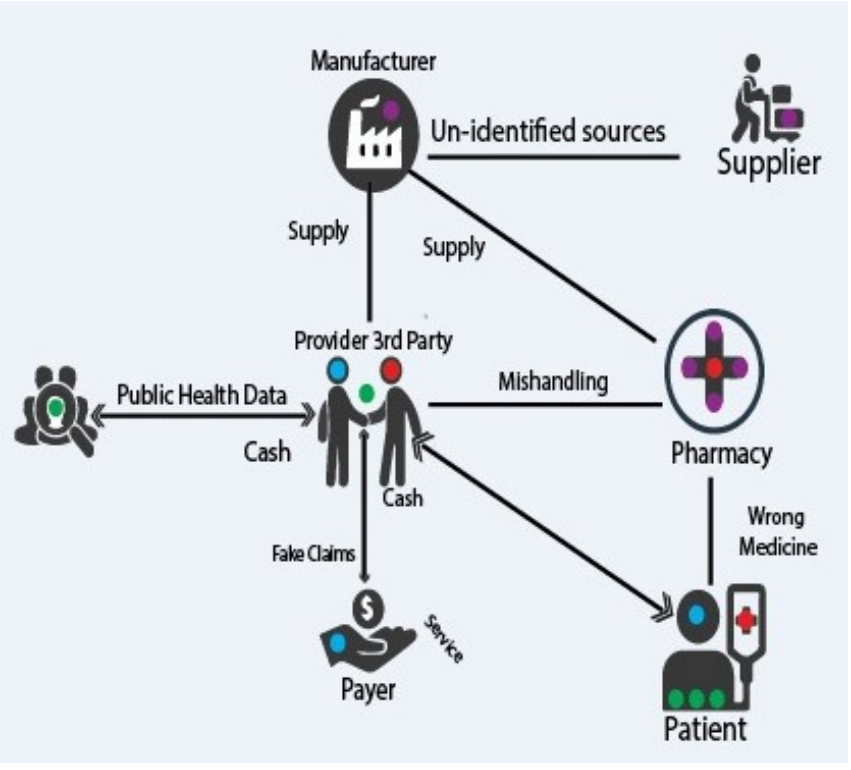

Fig. 2. Overview of the relationship among five major stakeholders in the healthcare sector (Suppliers, manufacturer, and pharmacies form a single unit of pharma supply chain)

Scenario 4. Research Organizations: Public health data is required by research organizations and pharmaceutical companies to track new diseases, invent their treatments and drug discovery. Health care data sharing is prerequisite for national data collection, clinical audit, and research [8]. Patient data recorded by a provider is shared with these organizations without the consent of patients, which is the violation of patient's privacy. Usually, patients are ignorant of the fact that their personal data is being shared with other parties without their consent which is the violation of patient's privacy and needed to be addressed [45]. Moreover, a chain of historical events describing exact switching points is crucial to track accelerated clinical trials and researches. Unavailability of data provenance contributes to undermining the reproducibility of outcomes. Maladministration lifts up the data snooping and misinterpreted reportage in disclosing the outcomes of trials in order to show the effects of new drugs discovered [23]. Patients' consent management, data management, data integrity, and transparent results are the major concerns of a trustful and productive system.

Scenario 5. Pharmaceutical Supply Chain Management: In the pharmaceutical supply chain, major players are suppliers, brokers, manufacturers, and pharmacies. It is a complex system including a diverse range of activities of acquisition of raw material, production, storage, distribution, etc. Proper management and monitoring are required to ensure reliability. Drug ingredients may travel through many sources where origin and quality of ingredients are not certified. Substandard constituents and illegitimate sources can play an important role in the production of counterfeit medicine [46]. So data provenance is obligatory to track genuine records, verified product information, and ownership in the drug supply chain. But current systems lack to maintain historical information to tackle the issues of counterfeit drugs [47].

Scenario 6. Prescription Management at Pharmacies:
Pharmacy is one of the supply chain stakeholders which forms a triangular relationship with provider and patient which is shown in Fig. 2 by using red color. Prescriptions forwarded by providers may be mishandled or un-deciphered at pharmacies and as a result, wrong medicine may be dispensed to a patient or it can also be given to an un-intended patient when prescriptions are jumbled up [48] [47]. The National Health Service (NHS)'s solution of electronic prescriptions rectifies many of the problems of the manual system. However, electronic prescriptions sent to community pharmacies from general practitioners expose patient confidentiality and need intense prescription management [49]. Moreover, main logistics inefficiencies executed by the hospital pharmacy may result in a big threat to the patients' safety. These inefficient activities may include incorrect inventory management, medicine shortage, long procurement cycles, time-consuming product recalls and improper use of technology [50]. Record management, data exchange among other supply chain stakeholders, security of records and privacy violation of patients' data are the major issues exhibited by this player of the healthcare sector.

In above-mentioned scenarios, some issues are observed to be common for all healthcare stakeholders. Major issues of each player are tabulated in Table III.

\section{B. RQ2: What Blockchain features are used to resolve the identified issues?}

Blockchain technologies have applicability in different scenarios which are the key benefit of this technology. The rapid advancement of healthcare sector can be envisioned by implementing the following features of blockchain technology, combating the major issues of the sector:

Distributed Digital Ledger resolves the issue of record management: Record management is inevitable to the success of every organization, which requires intense resource consumption in terms of human resources as well as software and hardware. Blockchain, the distributed ledger technology with no central authority promises to transform the current costly systems to inexpensive and easy to implement systems with higher efficiency and productivity [29] [17]. Decentralized communication among all medical stakeholders can be achieved as the same information is replicated throughout the permissioned nodes without any intermediary. Controlled access and real-time updates in records can increase fraud detection, combatting counterfeit drugs, claim adjudication and verified reproducibility in research [6]. The proposed work [45] can altogether lessen the turnaround time for EMR sharing intended for research and supervision of patient care, enhances basic management and reduces the general cost.

Interoperability resolves the issue of data exchange: Blockchain distributed ledger technology can resolve the issue of incompatible fragmented patient health records resulting in improved coordination and quality of care. This synchronized infrastructure will fasten real-time patients' notification, latest health and treatment information sharing and faster product innovation as the exchange of data will enhance market competitions positively [10] [51] [52] [38]. It helps in avoiding a single point of failure and it can facilitate in medical research by providing reliable data to different institutes so that better solution of patient care can be discovered [53]. Claim processing is usually affected by the complications of traditional 


\begin{tabular}{c|c|c|c|c|c}
\hline \multirow{2}{*}{ Issues } & \multicolumn{5}{c}{ Healthcare Stakeholders } \\
\cline { 2 - 6 } & Provider & Patient & Payer & Research Organizations & Supply chain \\
\hline Record Management & Yes & Yes & Yes & Yes & Yes \\
\hline Data Exchange & Yes & Yes & Yes & Yes & Yes \\
\hline Security & Yes & Yes & Yes & No & Yes \\
\hline Lack of Data Provenance & No & Yes & Yes & Yes & Yes \\
\hline Privacy & No & Yes & No & No & No \\
\hline Monetization & No & Yes & No & No &
\end{tabular}

distributed records, can be addressed by blockchain technology's interoperability feature [6] [46]. Shared immutable prescriptions can enhance medication reconciliation and quality of care.

Consensus mechanism and cryptography resolves the issue of security and privacy: Auditability and transparency is the magic demonstrated by immutable blockchain that has the ability to captivate the attention of users. Patient-controlled secure access is guaranteed by implementing consensus protocol and cryptographic keys in blockchain technology as the only private key can decrypt the data. Patient owns his/her data and also has the right to grant and revoke access to other persons (providers, payers or researchers) [6] [39] [17] [22] [5]. Security is one of the major issues encountered by almost every organization due to the reliance on a central authority. However, alteration in transactions in a blockchain based system will require the consensus of network and high computational power so data tampering is restricted.

Traceability and time stamping resolves the issue of lack of data provenance: Time stamped, verified records for claim qualification can save payers from hazardous financial loss. Findings of clinical trials require clarity, no data snooping; accurate endpoint switching, etc. These issues can be conquered by traceability feature of blockchain that provides the historical information by maintaining a chain of time-stamped blocks [23] [45] [22]. Drug manufacturing and distribution process can be tracked to detect counterfeit medicines using blockchain as it provides data provenance.

Digital Currency resolves the issue of monetization: In a blockchain based network, miners are rewarded with cryptocurrency as an incentive for consuming their computation powers and to serve and run the system. The cost has to be determined when a DApp provides services for patient and provider [28] [22]. Healthbank [8] has been providing a platform for patients and research organizations. They give financial rewards to the patient for their contributions and use health data for research and academic purposes.
C. RQ3: What are the challenges of Blockchain technology after its implementation in the Healthcare sector?

This new born technology has the potential to accelerate the healthcare sector however some implementation challenges are also discussed below:

Scalability: Blockchain powered healthcare system when uses sensor devices for patient care, faces storage limitation problem and requires heavy load computation that needs to be resolved [18]. Moreover, Blockchain network executes the transactions too slow and consequently system results in slow throughput as it may take days to complete a single transaction [54]. The transaction time is very long i.e. Bitcoin blockchain executes seven transactions per second (1 MB block size), depending upon the protocol (proof of work), in comparison with the Visa and Twitter networks, which perform 2000 and 5000 transactions per second respectively. Such a speed issue may limit the scalability of the blockchain network [6], [54]. All blocks are stored on every node which exists in the distributed system of the blockchain, also creates the issue of speed and scalability [07]. Real-time blockchain based health care applications may suffer from speed and scalability issue in a larger network.

Security (threat of $51 \%$ attack): Blockchain runs on consensus. Majority or more than half of total nodes i.e. $51 \%$, malicious miners may occupy the network and reject the blocks from other honest miners, their greater computation power may seize the precious information or coins [6] [54] [5]. However, there are fewer chances of this threat in a larger network.

Disclosure of confidentiality: Open source nature of the blockchain database pulls out another limitation which is "transparency discloses confidentiality". It is more critical for healthcare records of patients and biomedical applications because patients related records are highly sensitive [6]. (due to transparent nature of blockchain, healthcare or other's sensitive data are shown to everyone on the blockchain)

Anonymity and data privacy: Another challenge to blockchain is that the uncertainty and fraud hype surrounds it [20]. Criminals may use cryptocurrencies taking advantage of anonymity under the blockchain network. People may buy illegal drugs on the Dark web by using cryptocurrencies. Fraud 
hype is also associated with blockchain networks when hackers use "Ransomware" to seize computer networks and demand payment in cryptocurrencies [39]. Data privacy is compromised in a public blockchain network [55] [57] whereas patient is concerned about his/her privacy of health data. Moreover, blockchain based cloud environment exhibit limitations on access control methods for privacy and security of health data [56].

Environmental unsustainability: Environmentally unsustainability and inefficiency is another issue with current blockchain implementations. The "proof-of-work" requirements in current versions of the blockchain require massive amounts of electricity; the energy cost of a single Bitcoin transaction could power 1.5 American homes for a day [43]. As the ledgers get longer, the math gets harder, and the amount of power being used increases. Thankfully, there are less computing-intensive versions of the blockchain in development [39]. But the associated challenge of storage intensive medical records are needed to be resolved.

\section{Threats to VALIDity}

This SLR tries to compare and classify the blockchain technologies for the healthcare sector. Systematic literature reviews are considered reliable in general but this review can have some potential limitations. Expected limitations are restricted to related studies, identification and selection, insufficient data extraction, and unconcluded results.

\section{A. Threats to Identification and Selection of Primary Studies}

To provide a deeper insight to use blockchain in the healthcare sector, we try to gather as many primary studies as possible for extraction of knowledge to avoid biases. But as this new domain is in its early stages and researchers are exploring it very keenly and eagerly, so in the process of publication, many related research studies may become available in near future which is not presented here. A classification criterion is designed to shortlist and appropriately classifies as many primary studies as possible. We included all the related studies and did not evaluate these studies by giving quality scores.

\section{B. Threats to Data Extraction}

We gathered as many articles as possible related to our domain including primary and secondary articles, no quality score was assigned to select studies which help in prioritizing the result outcomes and research trends, is a major threat to data extraction. Another threat is that the data extracted from these articles based on our perspective of research questions and motivations. There are clear chances that readers and researchers may find some points that need to be considered to make this study more influential.

\section{Threats to Synthesis and Results}

As quality score is not assigned to collected studies it may lead to less quality results and synthesis.

\section{CONClusion AND Future Work}

Blockchain Technology is relatively new in the field of computing and healthcare as well. This technology has great potential in the sub-sectors of the healthcare field solving the major issues with its features and properties. Technology has the potential to revolutionize the whole ecosystem. Providers, patients, and research organizations are more focused on its initial journey and needs more research however the intense research work must be carried out in health insurance and pharmaceutical supply chains. Blockchain technology is also observed with some challenges while an implementation that needs to be solved with further research. Threats to the validity of our study discussed above may lead to better future work of this research work.

\section{REFERENCES}

[1] Zheng, Zibin, Shaoan Xie, Hongning Dai, Xiangping Chen, and Huaimin Wang. "An overview of blockchain technology: Architecture, consensus, and future trends." In 2017 IEEE International Congress on Big Data (BigData Congress), pp. 557-564. IEEE, 2017.

[2] Nakamoto, Satoshi. "Bitcoin: A peer-to-peer electronic cash system," http://bitcoin. org/bitcoin. pdf." (2008).

[3] Kuo, Tsung-Ting, Hyeon-Eui Kim, and Lucila Ohno-Machado. "Blockchain distributed ledger technologies for biomedical and health care applications." Journal of the American Medical Informatics Association 24, no. 6 (2017): 1211-1220.

[4] Daniel, Jeff, Arman Sargolzaei, Mohammed Abdelghani, Saman Sargolzaei, and Ben Amaba. "Blockchain Technology, Cognitive Computing, and Healthcare Innovations." Journal of Advances in Information Technology Vol 8, no. 3 (2017).

[5] Mettler, Matthias. "Blockchain technology in healthcare: The revolution starts here." In 2016 IEEE 18th International Conference on e-Health Networking, Applications and Services (Healthcom), pp. 1-3. IEEE, 2016.

[6] Rabah, Kefa. "Challenges \& opportunities for blockchain powered healthcare systems: A review." Mara Research Journal of Medicine \& Health Sciences-ISSN 2523-5680 1, no. 1 (2017): 45-52.

[7] Zhang, Peng, Michael A. Walker, Jules White, Douglas C. Schmidt, and Gunther Lenz. "Metrics for assessing blockchain-based healthcare decentralized apps." In 2017 IEEE 19th International Conference on e-Health Networking, Applications and Services (Healthcom), pp. 1-4. IEEE, 2017.

[8] Park, Jin, and Jong Park. "Blockchain security in cloud computing: Use cases, challenges, and solutions." Symmetry9, no. 8 (2017): 164.

[9] Ahram, Tareq, Arman Sargolzaei, Saman Sargolzaei, Jeff Daniels, and Ben Amaba. "Blockchain technology innovations." In 2017 IEEE Technology \& Engineering Management Conference (TEMSCON), pp. 137141. IEEE, 2017.

[10] Kuo, Tsung-Ting, Hugo Zavaleta Rojas, and Lucila Ohno-Machado. "Comparison of blockchain platforms: a systematic review and healthcare examples." Journal of the American Medical Informatics Association 26, no. 5 (2019): 462-478.

[11] Risius, Marten, and Kai Spohrer. "A blockchain research framework." Business \& Information Systems Engineering 59, no. 6 (2017): 385-409.

[12] 45. Hölbl, Marko, Marko Kompara, Aida Kamišalić, and Lili Nemec Zlatolas. "A systematic review of the use of blockchain in healthcare." Symmetry 10, no. 10 (2018): 470.

[13] Xia, Q. I., Emmanuel Boateng Sifah, Kwame Omono Asamoah, Jianbin Gao, Xiaojiang Du, and Mohsen Guizani. "MeDShare: Trust-less medical data sharing among cloud service providers via blockchain." IEEE Access 5 (2017): 14757-14767.

[14] Banerjee, Mandrita, Junghee Lee, and Kim-Kwang Raymond Choo. "A blockchain future for internet of things security: A position paper." Digital Communications and Networks 4, no. 3 (2018): 149-160.

[15] Swan, Melanie. Blockchain: Blueprint for a new economy. "O'Reilly Media, Inc.", 2015. 
[16] Zhang, Peng, Douglas C. Schmidt, Jules White, and Gunther Lenz. "Blockchain technology use cases in healthcare." In Advances in Computers, vol. 111, pp. 1-41. Elsevier, 2018.

[17] Dwivedi, Ashutosh Dhar, Gautam Srivastava, Shalini Dhar, and Rajani Singh. "A decentralized privacy-preserving healthcare blockchain for iot." Sensors 19, no. 2 (2019): 326.

[18] Peters, Gareth W., and Efstathios Panayi. "Understanding modern banking ledgers through blockchain technologies: Future of transaction processing and smart contracts on the internet of money." In Banking beyond banks and money, pp. 239-278. Springer, Cham, 2016.

[19] Mamoshina, Polina, Lucy Ojomoko, Yury Yanovich, Alex Ostrovski, Alex Botezatu, Pavel Prikhodko, Eugene Izumchenko et al. "Converging blockchain and next-generation artificial intelligence technologies to decentralize and accelerate biomedical research and healthcare." Oncotarget 9 , no. 5 (2018): 5665.

[20] Tama, Bayu Adhi, Bruno Joachim Kweka, Youngho Park, and KyungHyune Rhee. "A critical review of blockchain and its current applications." In 2017 International Conference on Electrical Engineering and Computer Science (ICECOS), pp. 109-113. IEEE, 2017.

[21] Nugent, Timothy, David Upton, and Mihai Cimpoesu. "Improving data transparency in clinical trials using blockchain smart contracts." F1000Research 5 (2016).

[22] Griggs, Kristen N., Olya Ossipova, Christopher P. Kohlios, Alessandro N. Baccarini, Emily A. Howson, and Thaier Hayajneh. "Healthcare blockchain system using smart contracts for secure automated remote patient monitoring." Journal of medical systems 42, no. 7 (2018): 130.

[23] Benchoufi, Mehdi, and Philippe Ravaud. "Blockchain technology for improving clinical research quality." Trials 18, no. 1 (2017): 335.

[24] Azaria, Asaph, Ariel Ekblaw, Thiago Vieira, and Andrew Lippman. "Medrec: Using blockchain for medical data access and permission management.” In 2016 2nd International Conference on Open and Big Data (OBD), pp. 25-30. IEEE, 2016.

[25] Xia, Qi, Emmanuel Sifah, Abla Smahi, Sandro Amofa, and Xiaosong Zhang. "BBDS: Blockchain-based data sharing for electronic medical records in cloud environments." Information 8, no. 2 (2017): 44.

[26] Yue, Xiao, Huiju Wang, Dawei Jin, Mingqiang Li, and Wei Jiang. "Healthcare data gateways: found healthcare intelligence on blockchain with novel privacy risk control." Journal of medical systems40, no. 10 (2016): 218.

[27] Zhang, Peng, Jules White, Douglas C. Schmidt, Gunther Lenz, and S. Trent Rosenbloom. "Fhirchain: applying blockchain to securely and scalably share clinical data." Computational and structural biotechnology journal 16 (2018): 267-278

[28] Angraal, Suveen, Harlan M. Krumholz, and Wade L. Schulz. "Blockchain technology: applications in health care." Circulation: Cardiovascular Quality and Outcomes 10, no. 9 (2017): e003800.

[29] Randall, David, Pradeep Goel, and Ramzi Abujamra. "Blockchain applications and use cases in health information technology." J Health Med Informat 8, no. 276 (2017): 2.

[30] Kitchenham, Barbara, O. Pearl Brereton, David Budgen, Mark Turner, John Bailey, and Stephen Linkman. "Systematic literature reviews in software engineering-a systematic literature review." Information and software technology 51, no. 1 (2009): 7-15.

[31] Khan, Muhammad Murad, Roliana Ibrahim, and Imran Ghani. "Cross domain recommender systems: a systematic literature review." ACM Computing Surveys (CSUR) 50, no. 3 (2017): 36.

[32] Yumna, Hafiza, Muhammad Murad Khan, Maria Ikram, and Sabahat Ilyas. "Use of Blockchain in Education: A Systematic Literature Review." In Asian Conference on Intelligent Information and Database Systems, pp. 191-202. Springer, Cham, 2019.

[33] Aly, Mohab, Foutse Khomh, Mohamed Haoues, Alejandro Quintero, and Soumaya Yacout. "Enforcing Security in Internet of Things Frameworks: A Systematic Literature Review." Internet of Things (2019): 100050.

[34] Alomar, Noura, Mansour Alsaleh, and Abdulrahman Alarifi. "Social authentication applications, attacks, defense strategies and future research directions: a systematic review." IEEE Communications Surveys \& Tutorials 19, no. 2 (2017): 1080-1111.

[35] Till, Brian M., Alexander W. Peters, Salim Afshar, and John G. Meara "From blockchain technology to global health equity: can cryptocurren- cies finance universal health coverage?." BMJ global health 2, no. 4 (2017): e000570.

[36] Anderson, James G. "Social, ethical and legal barriers to e-health." International journal of medical informatics 76, no. 5-6 (2007): 480-483.

[37] Lau, E. "Decoding the hype: Blockchain in Healthcare-A Software Architecture for the provision of a patient summary to overcome interoperability issues." Master's thesis, 2018.

[38] Liang, Xueping, Juan Zhao, Sachin Shetty, Jihong Liu, and Danyi Li. "Integrating blockchain for data sharing and collaboration in mobile healthcare applications." In 2017 IEEE 28th Annual International Symposium on Personal, Indoor, and Mobile Radio Communications (PIMRC), pp. 1-5. IEEE, 2017.

[39] Hoy, Matthew B. "An introduction to the blockchain and its implications for libraries and medicine." Medical reference services quarterly 36, no. 3 (2017): 273-279.

[40] Karafiloski, Elena, and Anastas Mishev. "Blockchain solutions for big data challenges: A literature review." In IEEE EUROCON 2017-17th International Conference on Smart Technologies, pp. 763-768. IEEE, 2017.

[41] Kaplan, Bonnie. "Selling health data: de-identification, privacy, and speech." Cambridge Quarterly of Healthcare Ethics 24, no. 3 (2015): 256-271.

[42] Skiba, Diane J. "The potential of Blockchain in education and health care.” Nursing education perspectives 38, no. 4 (2017): 220-221.

[43] Brodersen, C., B. Kalis, C. Leong, E. Mitchell, E. Pupo, A. Truscott, and L. Accenture. "Blockchain: Securing a New Health Interoperability Experience." Accenture LLP (2016).

[44] Engelhardt, Mark A. "Hitching healthcare to the chain: An introduction to blockchain technology in the healthcare sector." Technology Innovation Management Review 7, no. 10 (2017).

[45] Dubovitskaya, Alevtina, Zhigang Xu, Samuel Ryu, Michael Schumacher, and Fusheng Wang. "Secure and trustable electronic medical records sharing using blockchain." In AMIA Annual Symposium Proceedings, vol. 2017, p. 650. American Medical Informatics Association, 2017.

[46] Mackey, Tim K., and Gaurvika Nayyar. "A review of existing and emerging digital technologies to combat the global trade in fake medicines." Expert opinion on drug safety 16, no. 5 (2017): 587-602.

[47] Clauson, Kevin A., Elizabeth A. Breeden, Cameron Davidson, and Timothy K. Mackey. "Leveraging blockchain technology to enhance supply chain management in healthcare." Blockchain in Healthcare Today (2018).

[48] Huckvale, Christopher, Josip Car, Masanori Akiyama, Safurah Jaafar, Tawfik Khoja, Ammar Bin Khalid, Aziz Sheikh, and Azeem Majeed. "Information technology for patient safety." BMJ Quality \& Safety 19, no. Suppl 2 (2010): i25-i33.

[49] Porteous, Terry, Christine Bond, Roma Robertson, Philip Hannaford, and Ehud Reiter. "Electronic transfer of prescription-related information: comparing views of patients, general practitioners, and pharmacists." $\mathrm{Br}$ J Gen Pract 53, no. 488 (2003): 204-209.

[50] Romero, Alejandro. "Managing medicines in the hospital pharmacy: logistics inefficiencies." In Proceedings of the World Congress on Engineering and Computer Science, vol. 2, pp. 1-6. 2013.

[51] Raju, Saravanan, Vandita Rajesh, and Jitender S. Deogun. "The Case for a Data Bank: an Institution to Govern Healthcare and Education." In Proceedings of the 10th International Conference on Theory and Practice of Electronic Governance, pp. 538-539. ACM, 2017.

[52] Zhang, Peng, Jules White, Douglas C. Schmidt, and Gunther Lenz. "Design of blockchain-based apps using familiar software patterns with a healthcare focus." In Proceedings of the 24th Conference on Pattern Languages of Programs, p. 19. The Hillside Group, 2017.

[53] Kuo, Tsung-Ting, and Lucila Ohno-Machado. "Modelchain: Decentralized privacy-preserving healthcare predictive modeling framework on private blockchain networks." arXiv preprint arXiv:1802.01746 (2018).

[54] Lazar, Max A., Zihang Pan, Renee-Marie Ragguett, Yena Lee, Mehala Subramaniapillai, Rodrigo B. Mansur, Nelson Rodrigues, and Roger S. McIntyre. "Digital revolution in depression: a technologies update for clinicians." Personalized Medicine in Psychiatry 4 (2017): 1-6.

[55] Reyna, Ana, Cristian Martín, Jaime Chen, Enrique Soler, and Manuel Díaz. "On blockchain and its integration with IoT. Challenges and 
opportunities.” Future Generation Computer Systems 88 (2018): 173190.

[56] Esposito, Christian, Alfredo De Santis, Genny Tortora, Henry Chang, and Kim-Kwang Raymond Choo. "Blockchain: A panacea for healthcare cloud-based data security and privacy?.” IEEE Cloud Computing 5, no. 1 (2018): 31-37.
[57] Tasatanattakool, Pinyaphat, and Chian Techapanupreeda. "Blockchain: Challenges and applications." In 2018 International Conference on Information Networking (ICOIN), pp. 473-475. IEEE, 2018. 